\title{
DIRECT SUMS OF EXACT COVERS OF COMPLEXES
}

\author{
ALINA IACOB
}

\begin{abstract}
A ring $R$ is left noetherian if and only if the direct sum of injective envelopes of any family of left $R$-modules is the injective envelope of the direct sum of the given family of modules (or equivalently, if and only if the direct sum of any family of injective left $R$-modules is also injective). This result of Bass ([2]) led to a series of similar closure questions concerning classes of modules and classes of envelopes and covers (Chase in [4] considers the question of the closure of the class of flat modules with respect to products).

Motivated by Bass' result we consider the question of direct sums of exact covers of complexes. From the close connection between minimal injective resolutions of modules and exact covers of complexes it seemed reasonable to conjecture that we get this closure over left noetherian rings. In this paper we show that this is not the case and that under various additional hypotheses on the ring that in fact the ring must have finite left global dimension for this to happen. Our results raise what we consider an interesting question about characterizing the local rings of finite global dimension in terms of a certain property of minimal projective resolutions of finitely generated modules over the local ring.

We also consider the closely related question of when the direct sum of DG-injective complexes is DG-injective.
\end{abstract}

\section{Introduction}

In [6] it was proved that every complex $X$ of left $R$-modules (for any ring $R$ ) has an exact cover (see Section 2 for definitions).

As an example consider a minimal injective resolution

$$
0 \rightarrow M \rightarrow E^{0} \rightarrow E^{1} \rightarrow \ldots
$$

of any left $R$-module $M$. Then the obvious map of complexes

$$
\begin{aligned}
(\ldots \rightarrow 0 \rightarrow 0 \rightarrow 0 \rightarrow M & \left.\rightarrow E^{0} \rightarrow E^{1} \rightarrow \ldots\right) \rightarrow \\
(\ldots \rightarrow 0 \rightarrow 0 & \rightarrow 0 \rightarrow M \rightarrow 0 \rightarrow 0 \rightarrow \ldots)
\end{aligned}
$$

is an exact cover of $M$ considered as a complex concentrated at 0 .

From this example and the result of Bass ([2]) we quickly see that in order that the direct sum of exact covers to be an exact cover the ring must be left noetherian. So it seemed natural to conjecture that in fact this always is the case over left noetherian rings.

Received September 27, 2004. 
It is known that the kernel of any exact cover is a DG-injective complex (see below for definitions) and any DG-injective complex is the kernel of an exact precover ([6], Theorem 3.18 and Lemma 3.21). So a necessary condition in order that every direct sum of exact covers to be an exact cover is that every direct sum of DG-injective complexes be still DG-injective.

That the class of DG-injective complexes is not, in general, closed under direct sums can be seen from [8] (Example, pp. 68). If l.gl.dim $R<\infty$ then any complex $K$ of injective left $R$-modules is DG-injective (see [1], Proposition 3.4). Consequently, if $R$ is left noetherian and l.gl.dim $R<\infty$ then for any family $\left(K_{i}\right)_{i \in I}$ of DG-injective complexes we have that $\oplus_{i \in I} K_{i}$ is DGinjective. We give (Proposition 3 ) a necessary condition for a left noetherian ring $R$ in order that every direct sum of DG-injective complexes to be DGinjective. We use this result to prove (Theorem 2) that if $R$ is a commutative local Gorenstein ring then the following are equivalent:

(1) every direct sum of DG-injective complexes is DG-injective.

(2) gl.dim $R<\infty$.

Theorem 3 proves that (1) and (2) are equivalent for any commutative Gorenstein ring $R$.

Using this result we prove (Theorem 4 ) that if $R$ is a commutative Gorenstein ring then the following are equivalent:

(1) If $E_{i} \rightarrow X_{i}$ is an exact cover for any $i \in I$ then $\oplus_{i \in i} E_{i} \rightarrow \oplus_{i \in I} X_{i}$ is an exact cover.

(2) gl.dim $R<\infty$.

We consider then a complete commutative local noetherian ring $R$ such that every direct sum of DG-injective complexes over $R$ is DG-injective. Let $\ldots \rightarrow R^{\beta_{1}} \stackrel{f_{1}}{\longrightarrow} R^{\beta_{0}} \stackrel{f_{0}}{\longrightarrow} M \rightarrow 0$ be a minimal projective resolution of a finitely generated $R$-module $M$. Theorem 5 shows that for each $l \geq 1$ there is $\bar{n} \geq 1$ such that the entries of the matrix that represents $f_{n}$ are all in $m^{l}$, for any $n \geq \bar{n}$.

Theorem 6 shows that this result is true for any commutative local noetherian ring $(R, m, k)$ with the property that the direct sum of DG-injective complexes is DG-injective.

In particular, if $\ldots \rightarrow R^{\beta_{1}} \stackrel{f_{1}}{\longrightarrow} R^{\beta_{0}} \stackrel{f_{0}}{\longrightarrow} k \rightarrow 0$ is a minimal projective resolution and $A_{n}$ is the matrix that represents $f_{n}$ then, by Theorem 6, for each $l \geq 1$ there is $n_{l} \geq 1$ such that all entries of $A_{n}$ are in $m^{l}$, for any $n \geq n_{l}$. It is not known if this guarantees that there is $\bar{n} \geq 1$ such that $f_{n}=0$ for any $n \geq \bar{n}$, or equivalently gl.dim $R=\operatorname{proj} \operatorname{dim} k<\infty$. 
As a consequence of Theorem 6 we show that for a commutative local artinian ring $R$ a necessary and sufficient condition in order that every direct sum of DG-injective complexes to be DG-injective is that gl.dim $R<\infty$. Theorem 7 shows that the result holds for any commutative artinian ring $R$. We use Theorem 7 to prove that if $R$ is commutative artinian then any direct sum of exact covers of complexes of $R$-modules is still an exact cover if and only if gl.dim $R<\infty$ (Theorem 8 ).

\section{Preliminaries}

Let $R$ be any ring.

A (chain) complex $C$ of $R$-modules is a sequence $C=\ldots \rightarrow C_{2} \stackrel{\partial_{2}}{\longrightarrow}$ $C_{1} \stackrel{\partial_{1}}{\longrightarrow} C_{0} \stackrel{\partial_{0}}{\longrightarrow} C_{-1} \stackrel{\partial_{-1}}{\longrightarrow} C_{-2} \rightarrow \ldots$ of $R$-modules and $R$-homomorphisms such that $\partial_{n-1} \circ \partial_{n}=0$ for all $n \in Z$.

A chain complex of the form $C=\ldots \rightarrow C^{-2} \stackrel{\partial^{-2}}{\longrightarrow} C^{-1} \stackrel{\partial^{-1}}{\longrightarrow} C^{0} \stackrel{\partial^{0}}{\longrightarrow}$ $C^{1} \stackrel{\partial^{1}}{\longrightarrow} C^{2} \rightarrow \ldots$ is called a cochain complex. In this case $\partial^{n+1} \circ \partial^{n}=0$ for all $n \in \mathrm{Z}$. We note that a cochain complex is simply a chain complex with $C^{i}$ replaced by $C_{-i}$ and $\partial^{i}$ by $\partial_{-i}$.

Throughout the paper we use both the subscript notation for complexes and the superscript notation.

When we use superscripts for a complex we will use subscripts to distinguish complexes, for example $\left(K_{i}\right)_{i \in I}$ is a family of complexes and $K_{i}^{n}$ denotes the degree $n$ term of the complex $K_{i}$.

If $X$ and $Y$ are both complexes of left $R$-modules then $\mathscr{H o m}(X, Y)$ denotes the complex with $\mathscr{H} o m(X, Y)_{n}=\Pi_{q=p+n} \operatorname{Hom}_{R}\left(X_{p}, Y_{q}\right)$ and with differential given by $\partial(f)=\partial \circ f-(-1)^{n} f \circ \partial$, for $f \in \mathscr{H} \circ o m(X, Y)_{n}$.

Definition 1 ([6]). A complex $I$ is DG-injective if each $I^{n}$ is injective and if $\mathscr{C o m}(E, I)$ is exact for any exact complex $E$.

Recall that a complex $I$ is $K$-injective if for every exact complex $E$ the complex $\mathscr{H} o m(E, I)$ is exact ([10], Definition 1.1). Thus a complex $I$ is DGinjective if and only if each $I^{n}$ is injective and $I$ is $K$-injective in the sense of Spaltenstein.

It is known that if $I=\ldots \rightarrow 0 \rightarrow 0 \rightarrow I^{n_{0}} \rightarrow I^{n_{0}+1} \rightarrow I^{n_{0}+2} \rightarrow \ldots$ and each $I^{n}$ is injective then $I$ is DG-injective ([1], Remark 1.1.1).

If 1. gl.dim $R<\infty$ then any complex $I$ with all $I^{n}$ injective is DG-injective ([1], Proposition 3.4).

Throughout this paper $\operatorname{Hom}(X, Y)$ denotes the set of morphisms from $X$ to $Y$ in the category of complexes, and $\operatorname{Ext}^{i}(X, Y)$ are the right derived functors of $\operatorname{Hom}(-,-)$. 
Definition 2. A complex $E$ is injective if the functor $\operatorname{Hom}(-, E)$ is right exact.

If $M$ is an injective $R$-module then the complex $\ldots \rightarrow 0 \rightarrow M \stackrel{\text { id }}{\longrightarrow} M \rightarrow$ $0 \rightarrow \ldots$ (with the first $M$ in the $n$th place) is injective. In fact any injective complex is uniquely up to isomorphism the direct sum of such complexes (one such complex for each $n \in \mathbf{Z}$ ).

Proposition 1 ([6], Proposition 3.4). A complex I is DG-injective if and only if $\operatorname{Ext}^{1}(E, I)=0$ for any exact complex $E$.

Definition 3 ([6], pp. 35). A DG-injective complex $I=\ldots \rightarrow I^{n-1} \stackrel{g_{n-1}}{\longrightarrow}$ $I^{n} \stackrel{g_{n}}{\longrightarrow} I^{n+1} \rightarrow \ldots$ is said to be minimal DG-injective if for each $n$, Ker $g_{n}$ is essential in $I^{n}$.

Proposition 2 ([6], Proposition 3.16). A DG-injective complex is the direct sum of an injective complex and a minimal DG-injective complex. This direct sum decomposition is unique up to isomorphism.

Definition 4 ([6]). A morphism of complexes $\Phi: E \rightarrow X$ is an exact precover of $X$ if $E$ is exact and if $\operatorname{Hom}(F, E) \rightarrow \operatorname{Hom}(F, X)$ is surjective for any exact complex $F$.

If, moreover, any $f: E \rightarrow E$ such that $\Phi=\Phi \circ f$ is an automorphism of $E$, then $\Phi: E \rightarrow X$ is called an exact cover of $X$.

Theorem 1 ([6], Theorem 3.18). Every complex $X$ has an exact cover $E \rightarrow X$. A morphism $E \rightarrow X$ of complexes is an exact cover of $X$ if and only if $E$ is exact, $E \rightarrow X$ is surjective and $\operatorname{Ker}(E \rightarrow X)$ is a minimal DGinjective complex. If $E \rightarrow X$ is an exact cover, $E$ is injective if and only if $X$ is DG-injective.

We recall that for any $n$, and for any complex $X, X[n]$ denotes the complex such that $X[n]^{m}=X^{n+m}$ and whose boundary operators are $(-1)^{n} \partial^{n+m}$.

If $f: X \rightarrow Y$ is a morphism of complexes then there is an exact sequence $0 \rightarrow Y \rightarrow M(f) \rightarrow X[1] \rightarrow 0$ with $M(f)$ the associated mapping cone $\left(M(f)^{n}=X^{n+1} \oplus Y^{n}\right.$ and $\partial(x, y)=(-\partial x, f(x)+\partial y)$ for $(x, y) \in X^{n+1} \oplus$ $\left.Y^{n}\right)$.

Lemma 1 ([6], Lemma 3.21). Let I be a DG-injective complex and let $\mathrm{Id}: I \rightarrow I$ give the exact sequence $0 \rightarrow I \rightarrow M(\mathrm{Id}) \rightarrow I[1] \rightarrow 0$. Then $M(\mathrm{Id})$ is injective and $M(\mathrm{Id}) \rightarrow I[1]$ is an exact precover. If I is minimal then $I \rightarrow M(\mathrm{Id})$ is an injective envelope and $M(\mathrm{Id}) \rightarrow I[1]$ is an exact cover.

LEMma 2. If $\left(K_{n}\right)_{n \geq 0}$ is a family of DG-injective complexes then $\Pi_{n=0}^{\infty} K_{n}$ is DG-injective. 
Proof. Let $M$ be an exact complex.

Each $K_{n}$ is DG-injective, so by Proposition 1 we have $\operatorname{Ext}^{1}\left(M, K_{n}\right)=0$ for any $n \geq 0$.

Since $\operatorname{Ext}^{1}\left(M, \Pi_{n=0}^{\infty} K_{n}\right) \simeq \Pi_{n=0}^{\infty} \operatorname{Ext}^{1}\left(M, K_{n}\right)=0$ for any exact complex $M$ it follows (by Proposition 1) that $\prod_{n=0}^{\infty} K_{n}$ is DG-injective.

\section{A necessary condition in order that every direct sum of DG- injective complexes to be DG-injective}

Our first result in this section gives a necessary condition in order that every direct sum of DG-injective complexes to be DG-injective.

We recall that a morphism $f: X \rightarrow Y$ of complexes is called a quasiisomorphism if the maps $H^{n}(X) \rightarrow H^{n}(Y)$ are all isomorphisms.

Proposition 3. Let $R$ be a left noetherian ring. A necessary condition in order that the direct sum of DG-injective complexes to be DG-injective is that

$$
\frac{\prod_{n=0}^{\infty} C_{n}}{\oplus_{n=0}^{\infty} C_{n}}
$$

is injective, where $C_{n}$ is the $n$th cosyzygy of some module $C$.

Proof. Let $0 \rightarrow C \rightarrow E^{0} \rightarrow E^{1} \rightarrow E^{2} \rightarrow \ldots$ be an injective resolution of $C$.

Let

$$
\begin{aligned}
& K_{0}=\ldots \rightarrow 0 \rightarrow 0 \rightarrow 0 \rightarrow E^{0} \rightarrow E^{1} \rightarrow E^{2} \rightarrow \ldots \\
& K_{1}=\ldots \rightarrow 0 \rightarrow 0 \rightarrow E^{0} \rightarrow E^{1} \rightarrow E^{2} \rightarrow E^{3} \rightarrow \ldots \\
& K_{2}=\ldots \rightarrow 0 \rightarrow E^{0} \rightarrow E^{1} \rightarrow E^{2} \rightarrow E^{3} \rightarrow E^{4} \rightarrow \ldots
\end{aligned}
$$

Each $K_{n}$ is a bounded below complex of injective modules, so $K_{n}$ is DGinjective for any $n \geq 0$.

We show first that the inclusion map $\oplus_{n=0}^{\infty} K_{n} \stackrel{\psi}{\longrightarrow} \Pi_{n=0}^{\infty} K_{n}$ is a quasiisomorphism.

We have that

$$
H\left(\oplus_{n=0}^{\infty} K_{n}\right)=\oplus_{n=0}^{\infty} H\left(K_{n}\right) \quad \text { and } \quad H\left(\Pi_{n=0}^{\infty} K_{n}\right)=\Pi_{n=0}^{\infty} H\left(K_{n}\right)
$$

But each $K_{n}$ has at most one non-zero homology module and this is in the $n$th position. So we see that for each $l$

$$
\left(\oplus_{n=0}^{\infty} H\left(K_{n}\right)\right)^{l}=H\left(K_{l}\right) \quad \text { and } \quad\left(\Pi_{n=0}^{\infty} H\left(K_{n}\right)\right)^{l}=H\left(K_{l}\right)
$$


Hence we see that

$$
\oplus_{n=0}^{\infty} K_{n} \rightarrow \Pi_{n=0}^{\infty} K_{n}
$$

is a quasi-isomorphism.

- We show now that a necessary condition for $\oplus_{n=0}^{\infty} K_{n}$ to be DG-injective is that

$$
\frac{\Pi_{n=0}^{\infty} C_{n}}{\oplus_{n=0}^{\infty} C_{n}}
$$

is an injective module, where $C_{n}$ is the $n$th cosyzygy of $C$.

Let $E=\frac{\prod_{n=0}^{\infty} K_{n}}{\oplus_{n=0}^{\infty} K_{n}}$. Since the sequence $0 \rightarrow \oplus_{n=0}^{\infty} K_{n} \stackrel{\psi}{\longrightarrow} \prod_{n=0}^{\infty} K_{n} \rightarrow E \rightarrow$ 0 is exact and $\psi$ is a quasi-isomorphism is follows that $E$ is an exact complex.

If $\oplus_{n=0}^{\infty} K_{n}$ is DG-injective then since $\Pi_{n=0}^{\infty} K_{n}$ is also DG-injective (Lemma 2 ) it follows that $E$ is DG-injective ([6], Remark pp. 31).

Since $E$ is exact and DG-injective it follows ([6] Proposition 3.7) that $E$ is an injective complex.

We use the notation $K_{n}=\ldots \rightarrow K_{n}^{2} \stackrel{\alpha_{n}^{2}}{\longrightarrow} K_{n}^{1} \stackrel{\alpha_{n}^{1}}{\longrightarrow} K_{n}^{0} \rightarrow \ldots$ for any $n \geq 0$.

We have

$$
E=\ldots \rightarrow \frac{\Pi_{n=0}^{\infty} K_{n}^{2}}{\bigoplus_{n=0}^{\infty} K_{n}^{2}} \stackrel{g^{2}}{\longrightarrow} \frac{\Pi_{n=0}^{\infty} K_{n}^{1}}{\bigoplus_{n=0}^{\infty} K_{n}^{1}} \stackrel{g^{1}}{\longrightarrow} \frac{\Pi_{n=0}^{\infty} K_{n}^{0}}{\bigoplus_{n=0}^{\infty} K_{n}^{0}} \stackrel{g^{0}}{\longrightarrow} \frac{\prod_{n=0}^{\infty} K_{n}^{-1}}{\bigoplus_{n=0}^{\infty} K_{n}^{-1}} \rightarrow \ldots
$$

The complex $E$ is injective, so Ker $g^{n}$ is an injective $R$-module for any $n \in \mathbf{Z}$ ([8], Theorem 3.1.3). In particular Ker $g^{0}$ is injective.

- We show that $\operatorname{Ker} g^{0} \simeq \frac{\prod_{n=0}^{\infty} C_{n}}{\Theta_{n=0}^{\infty} C_{n}}$ (with $C_{n}$ the $n$th cosyzygy of $C$ ).

$g^{0}\left(\left(x_{n}\right)_{n \geq 0}+\oplus_{n=0}^{\infty} K_{n}^{0}\right)=\left(\alpha_{n}^{0}\left(x_{n}\right)\right)_{n \geq 0}+\oplus_{n=0}^{\infty} K_{n}^{-1}$.

If $\left(x_{n}\right)_{n \geq 0}+\oplus_{n=0}^{\infty} K_{n}^{0} \in \operatorname{Ker} g^{0}$ then $\left(\alpha_{n}^{0}\left(x_{n}\right)\right)_{n} \in \oplus_{n=0}^{\infty} K_{n}^{-1}$.

So $\alpha_{n}^{0}\left(x_{n}\right)=0 \Leftrightarrow x_{n} \in \operatorname{Ker} \alpha_{n}^{0}=\operatorname{Ker}\left(E^{n} \rightarrow E^{n+1}\right)=C_{n}$, for all but finitely many $n \geq 0$.

Let $y=\left(y_{n}\right)_{n \geq 0}$ with

$$
y_{n}= \begin{cases}x_{n}, & \text { if } x_{n} \in \operatorname{Ker} \alpha_{n}^{0} \\ 0, & \text { if } x_{n} \notin \operatorname{Ker} \alpha_{n}^{0}\end{cases}
$$

Then $y \in \Pi_{n=0}^{\infty} \operatorname{Ker} \alpha_{n}^{0}=\Pi_{n=0}^{\infty} C_{n}$.

Let $z=\left(x_{n}-y_{n}\right)_{n \geq 0}$. Then $z \in \oplus_{n=0}^{\infty} K_{n}^{0}=\oplus_{n=0}^{\infty} E^{n}$ and $\left(x_{n}\right)_{n \geq 0}=y+z \in$ $\Pi_{n=0}^{\infty} C_{n}+\oplus_{n=0}^{\infty} E^{n}$.

So

$$
\operatorname{Ker} g^{0} \subset \frac{\prod_{n=0}^{\infty} C_{n}+\oplus_{n=0}^{\infty} E^{n}}{\oplus_{n=0}^{\infty} E^{n}}
$$


If

$$
x+\oplus_{n=0}^{\infty} K_{n}^{0} \in \frac{\prod_{n=0}^{\infty} C_{n}+\oplus_{n=0}^{\infty} E^{n}}{\oplus_{n=0}^{\infty} E^{n}}=\frac{\prod_{n=0}^{\infty} \operatorname{Ker} \alpha_{n}^{0}+\oplus_{n=0}^{\infty} K_{n}^{0}}{\oplus_{n=0}^{\infty} K_{n}^{0}}
$$

then $x=y+z$ with $y \in \Pi_{n=0}^{\infty} \operatorname{Ker} \alpha_{n}^{0}$ and $z \in \oplus_{n=0}^{\infty} K_{n}^{0}$.

So $x+\oplus_{n=0}^{\infty} K_{n}^{0}=y+\oplus_{n=0}^{\infty} K_{n}^{0}$. Since $g^{0}\left(y+\oplus_{n=0}^{\infty} K_{n}^{0}\right)=\left(\alpha_{n}^{0}\left(y_{n}\right)\right)_{n \geq 0}+$ $\oplus_{n=0}^{\infty} K_{n}^{-1}=0+\oplus_{n=0}^{\infty} K_{n}^{-1}$ it follows that

$$
x+\oplus_{n=0}^{\infty} K_{n}^{0} \in \operatorname{Ker} g^{0}
$$

By (1) and (2) we have:

$$
\text { Ker } g^{0}=\frac{\Pi_{n=0}^{\infty} C_{n}+\oplus_{n=0}^{\infty} E^{n}}{\oplus_{n=0}^{\infty} E^{n}} \simeq \frac{\prod_{n=0}^{\infty} C_{n}}{\prod_{n=0}^{\infty} C_{n} \cap \oplus_{n=0}^{\infty} E^{n}}=\frac{\prod_{n=0}^{\infty} C_{n}}{\bigoplus_{n=0}^{\infty} C_{n}}
$$

So $\frac{\prod_{n=0}^{\infty} C_{n}}{\oplus_{n=0}^{\infty} C_{n}}$ is an injective module.

Another useful result is the following.

Lemma 3. Let $(R, m, k)$ be a commutative local noetherian ring and let $A$ be an artinian module. If $0 \rightarrow A \rightarrow E^{0} \rightarrow E^{1} \rightarrow E^{2} \rightarrow \ldots$ is a minimal injective resolution of $A$ then each $E^{n}$ is a finite direct sum of copies of $E(k)$.

Proof. Since $A$ is artinian, we have $A \subset E(k)^{n}$, for some $n \geq 1$ ([5], Theorem 3.4.3). So $E^{0}$ is a direct summand of $E(k)^{n}$. Therefore $E^{0}=E(k)^{\beta_{0}}$, with $1 \leq \beta_{0} \leq n$. If $K_{1}=\operatorname{Ker}\left(E^{1} \rightarrow E^{2}\right)$ then $0 \rightarrow A \rightarrow E^{0} \rightarrow K_{1} \rightarrow 0$ is exact. Since $E^{0}$ is artinian it follows that $K_{1}$ is artinian. So $K_{1} \subset E(k)^{l}$ for some $l \geq 1$. Then $E^{1}$ is a direct summand of $E(k)^{l}$. So $E^{1}=E(k)^{\beta_{1}}$, with $\beta_{1} \leq l$. Similarly, $E^{n}$ is a finite direct sum of copies of $E(k)$, for any $n \geq 0$.

Using Lemma 3 we can prove the following result.

Lemma 4. Let $(R, m, k)$ be a commutative local noetherian ring. Let $0 \rightarrow$ $k \rightarrow E(k) \stackrel{f_{0}}{\longrightarrow} E^{1} \stackrel{f_{1}}{\longrightarrow} E^{2} \rightarrow \ldots$ be a minimal injective resolution of $k$ and let $K_{n}=\operatorname{Ker}\left(E^{n} \rightarrow E^{n+1}\right)$ for any $n \geq 1$.

$K_{n}$ is injective if and only if for every homomorphism $f: k \rightarrow K_{n}$ there is a homomorphism $u: E(k) \rightarrow K_{n}$ such that $\left.u\right|_{k}=f$.

Proof. " $\Leftarrow$ " The injective envelope of $K_{n}$ is $E^{n}$. By Lemma $3 E^{n}=$ $\oplus_{i \in I} E_{i}$ with $E(k) \stackrel{\psi_{i}}{\simeq} E_{i}, \forall i \in I$.

Let $S_{i}=\psi_{i}(k) \simeq k$. Then $S_{i} \subset E_{i}$ is an injective envelope, $\forall i \in I$. Since $\psi_{i}(k) \cap K_{n} \leq \psi_{i}(k)$ and $\psi_{i}(k)$ is simple we have $\psi_{i}(k) \cap K_{n}=0$ or $\psi_{i}(k) \cap K_{n}=\psi_{i}(k)$. Since $K_{n}$ is essential in $E^{n}, \psi_{i}(k) \cap K_{n}=0$ would imply that $\psi_{i}(k)=0 \Leftrightarrow k=0$. False. 
So $\psi_{i}(k) \cap K_{n}=\psi_{i}(k) \Leftrightarrow S_{i}=\psi_{i}(k) \subset K_{n}$.

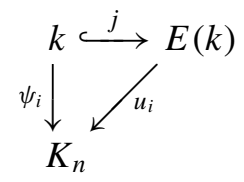

By hypothesis there exists $u_{i} \in \operatorname{Hom}\left(E(k), K_{n}\right)$ such that $u_{i} \circ j=\psi_{i}, \forall i \in I$.

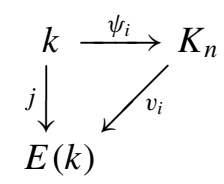

Since $\psi_{i}$ is an injection and $E(k)$ is an injective module there exists $v_{i} \in$ $\operatorname{Hom}\left(K_{n}, E(k)\right)$ such that $v_{i} \circ \psi_{i}=j$.

Then $v_{i} \circ u_{i} \circ j=v_{i} \circ \psi_{i}=j$. Since $j: k \rightarrow E(k)$ is an injective envelope it follows that $v_{i} \circ u_{i} \in$ Aut $E(k)$. Consequently, $u_{i}$ is an injection.

So $M_{i}=u_{i}(E(k)) \simeq E(k)$ is an injective module, $\forall i \in I$.

Since $u_{i}(k)=\psi_{i}(k)=S_{i} \subset M_{i} \simeq E(k)$ and $S_{i} \simeq k$ we have that $S_{i} \subset M_{i}$ is an injective envelope. So $M_{i}=E_{i} \forall i \in I$.

Since $E_{i} \subset K_{n} \forall i \in I$ we have $\oplus_{i \in I} E_{i} \subset K_{n} \Leftrightarrow E^{n} \subset K_{n} \subset E^{n} \Leftrightarrow$ $K_{n}=E^{n}$. So $K_{n}$ is an injective module.

“ $\Rightarrow$ " Let $f \in \operatorname{Hom}\left(k, K_{n}\right)$.

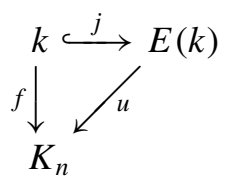

Since $K_{n}$ is injective and $j: k \rightarrow E(k)$ is an injection there is $u \in \operatorname{Hom}(E(k)$, $K_{n}$ ) such that $u \circ j=f$.

\section{Direct sums of exact covers over commutative Gorenstein rings}

We prove in this section that the class of DG-injective complexes over a commutative Gorenstein ring $R$ is closed under direct sums if and only if gl.dim $R<\infty$. Using this result we prove that if $R$ is a commutative Gorenstein ring then every direct sum of exact covers is an exact cover if and only if gl.dim $R<\infty$.

We start with the following result.

THEOREM 2. Let $R$ be a commutative local Gorenstein ring with maximal ideal $m$ and residue field $k$. The following are equivalent:

(1) Every direct sum of DG-injective complexes is DG-injective. 
(2) $\operatorname{gl} \operatorname{dim} R<\infty$.

Proof. (1) $\Rightarrow$ (2) Suppose gl.dim $R=\infty$. $R$ is a local $\operatorname{ring}$, so gl.dim $R=$ $\operatorname{proj} \operatorname{dim} k$ ([11], Corollary 4.4.12). Since $R$ is a Gorenstein ring, and proj $\operatorname{dim} k=\infty$ we have inj $\operatorname{dim} k=\infty$ ([5], Proposition 9.1.7).

Let $0 \rightarrow k \rightarrow E(k) \rightarrow E^{1} \rightarrow E^{2} \rightarrow \ldots$ be a minimal injective resolution of $k$. Let $K_{n}=\operatorname{Ker}\left(E^{n} \rightarrow E^{n+1}\right.$ ) for any $n \geq 0$ (with $K_{0}=k$ ). Since $\operatorname{inj} \operatorname{dim} k=\infty$ it follows that $K_{n}$ is not injective for any $n \geq 0$. By Lemma 4 this means that for each $n \geq 0$ there is $f_{n} \in \operatorname{Hom}\left(k, K_{n}\right)$ that can not be extended to a homomorphism $E(k) \rightarrow K_{n}$.

Let $f_{n}(1+m)=x_{n}$.

We know (Proposition 3) that if every direct sum of DG-injective complexes is DG-injective then $\frac{\prod_{n=0}^{\infty} C_{n}}{\oplus_{n=0}^{\infty} C_{n}}$ is an injective module where $C_{n}$ is the $n$th cosyzygy of some module $C$.

$R$ is a Gorenstein ring, so Gor inj $\operatorname{dim} k=d<\infty$.

Then $K_{n}$ is a Gorenstein injective module for any $n \geq d$ ([5], Proposition 11.2.5 and Theorem 10.1.4).

Let $C=K_{d}$ and let $M=\frac{\prod_{n=0}^{\infty} C_{n}}{\Theta_{n=0}^{\infty} C_{n}}=\frac{\prod_{n=d}^{\infty} K_{n}}{\Theta_{n=d}^{\infty} K_{n}}$.

Let $f: k \rightarrow M$ be defined by $f(1+m)=x$ with $x=\left(x_{n}\right)_{n \geq d}+\oplus_{n=d}^{\infty} K_{n}$.

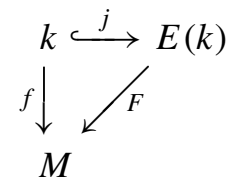

Since $M$ is an injective module and $j$ is an injection there is $F \in \operatorname{Hom}(E(k), M)$ such that $F \circ j=f$.

The sequence $0 \rightarrow \oplus_{n=d}^{\infty} K_{n} \rightarrow \prod_{n=d}^{\infty} K_{n} \stackrel{P}{\longrightarrow} M \rightarrow 0$ is exact, so we have an associated long exact sequence $0 \rightarrow \operatorname{Hom}\left(E(k), \oplus_{n=d}^{\infty} K_{n}\right) \rightarrow$ $\operatorname{Hom}\left(E(k), \Pi_{n=d}^{\infty} K_{n}\right) \rightarrow \operatorname{Hom}(E(k), M) \rightarrow \operatorname{Ext}^{1}\left(E(k), \oplus_{n=d}^{\infty} K_{n}\right)=0$ (since $\oplus_{n=d}^{\infty} K_{n}$ is Gorenstein injective and $E(k)$ is injective).

Since $0 \longrightarrow \operatorname{Hom}\left(E(k), \oplus_{n=d}^{\infty} K_{n}\right) \longrightarrow \operatorname{Hom}\left(E(k), \Pi_{n=d}^{\infty} K_{n}\right) \longrightarrow$ $\operatorname{Hom}(E(k), M) \rightarrow 0$ is exact there is $u \in \operatorname{Hom}\left(E(k), \prod_{n=d}^{\infty} K_{n}\right)$ such that $F=P \circ u$ (with $\left.P: \Pi_{n=d}^{\infty} K_{n} \rightarrow M, P\left(\left(z_{n}\right)_{n \geq d}\right)=\left(z_{n}\right)_{n \geq d}+\oplus_{n=d}^{\infty} K_{n}\right)$.

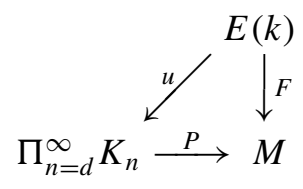

Let $u(1+m)=\left(y_{n}\right)_{n \geq d}$. Then $F(1+m)=P(u(1+m))=\left(y_{n}\right)_{n \geq d}+$ $\oplus_{n=d}^{\infty} K_{n}$. 
Since $\left.F\right|_{k}=f$, we have $F(1+m)=f(1+m)=\left(x_{n}\right)_{n \geq d}+\oplus_{n=d}^{\infty} K_{n}$. So $x_{n}=y_{n}$ for all but finitely many $n \geq d$.

Let $n \geq d$ be such that $x_{n}=y_{n}$.

Let $\pi_{n}: \Pi_{j=d}^{\infty} K_{j} \rightarrow K_{n}, \pi_{n}\left(\left(z_{j}\right)_{j \geq d}\right)=z_{n}$.

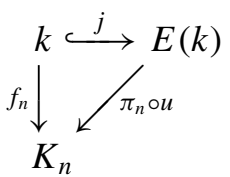

We have $\pi_{n} \circ u(1+m)=\pi_{n}\left(\left(y_{j}\right)_{j \geq d}\right)=y_{n}=x_{n}=f_{n}(1+m)$.

So $f_{n}: k \rightarrow K_{n}$ can be extended to a morphism $E(k) \rightarrow K_{n}$ for infinitely many $n \geq d$. Contradiction.

Hence gl.dim $R<\infty$.

(2) $\Rightarrow$ (1) Since gl.dim $R<\infty$ it follows that a complex $J$ is DG-injective if and only if each $J^{n}$ is an injective module.

Let $\left(J_{i}\right)_{i \in I}$ be a family of DG-injective complexes.

Since $R$ is noetherian and $J_{i}^{n}$ is injective $\forall i \in I$ it follows that $\oplus_{i \in I} J_{i}^{n}$ is injective. So $\bigoplus_{i \in I} J_{i}$ is a DG-injective complex.

In order to prove that the conditions (1) and (2) from Theorem 2 are in fact equivalent for any commutative Gorenstein ring we use the following well known result.

Lemma 5. Let $R$ be a commutative noetherian ring and let $S \subset R$ be a multiplicative set $(0 \notin S)$. If $E$ is an $S^{-1} R$ module then $E$ is an injective $S^{-1} R$-module if and only if $E$ is an injective $R$-module.

Theorem 3. Let $R$ be a commutative Gorenstein ring. The following are equivalent:

(1) Every direct sum of DG-injective complexes is DG-injective.

(2) gl.dim $R<\infty$.

Proof. (1) $\Rightarrow$ (2) We prove first that if every direct sum of DG-injectives is DG-injective then gl.dim $R_{p}<\infty$ for any $p \in \operatorname{Spec} R$.

Suppose there is $p \in \operatorname{Spec} R$ such that gl.dim $R_{p}=\infty$.

$R_{p}$ is a local ring with maximal ideal $p R_{p}$ and residue field $R_{p} / p R_{p}$ which is denoted $k(p)$. Since $R$ is a Gorenstein ring it follows that $R_{p}$ is also a Gorenstein ring ([5], Remark 2.3.8, and [3] Corollary 2.3).

Since proj $\operatorname{dim}_{R_{p}} k(p)=\mathrm{gl}$.dim $R_{p}=\infty$ and $R_{p}$ is Gorenstein, it follows that inj $\operatorname{dim}_{R_{p}} k(p)=\infty$ (by [5], Proposition 9.1.7).

Let $0 \rightarrow k(p) \rightarrow E_{p}^{0} \rightarrow E_{p}^{1} \rightarrow \ldots$ be a minimal injective resolution of $R_{p} k(p)$. Let $K_{p}^{n}=\operatorname{Ker}\left(E_{p}^{n} \rightarrow E_{p}^{n+1}\right)$. 
Since inj $\operatorname{dim}_{R_{p}} k(p)=\infty$ it follows that $K_{p}^{n}$ is not injective, $\forall n \geq 0$.

$R_{p}$ is Gorenstein, so Gor $\operatorname{inj} \operatorname{dim} k(p)=d_{p}<\infty$. Then $K_{p}^{n}$ is Gorenstein injective, $\forall n \geq d_{p}$.

Each $E_{p}^{i}$ is an injective $R_{p}$-module, therefore an injective $R$-module (Lemma 5). So each of the complexes:

$$
\begin{aligned}
& J_{d_{p}}=\ldots \rightarrow 0 \rightarrow 0 \rightarrow E_{p}^{0 \text { th }} \rightarrow E_{p}^{d_{p}^{d_{p}+1}} \rightarrow E_{p}^{d_{p}+2} \rightarrow \ldots \\
& J_{d_{p}+1}=\ldots \rightarrow 0 \rightarrow E_{p}^{d_{p}} \rightarrow E_{p}^{d_{p}+1} \rightarrow E_{p}^{d_{p}+2} \rightarrow E_{p}^{d_{p}+3} \rightarrow \ldots
\end{aligned}
$$

is DG-injective over $R$.

By hypothesis $\oplus_{n=d_{p}}^{\infty} J_{n}$ is a DG-injective complex over $R$.

Let $0 \rightarrow \oplus_{n=d_{p}}^{\infty} J_{n} \stackrel{\psi}{\longrightarrow} \Pi_{n=d_{p}}^{\infty} J_{n} \rightarrow E \rightarrow 0$ be exact.

Since $\psi$ is a homology isomorphism (same argument as in the proof of Proposition 3) it follows that $E$ is exact.

Both $\oplus_{n=d_{p}}^{\infty} J_{n}$ and $\Pi_{n=d_{p}}^{\infty} J_{n}$ are DG-injective, so $E$ is DG-injective ([6], Remark pp. 31). By [6], Proposition 3.7, $E$ is an injective complex. By [8], Theorem 3.1.3, $\operatorname{Ker}\left(E^{n} \rightarrow E^{n+1}\right)$ is injective, $\forall n \in \mathbf{Z}$.

In particular, $\operatorname{Ker}\left(E^{0} \rightarrow E^{1}\right)$ is an injective $R$-module. We showed (proof of Proposition 3) that $\operatorname{Ker}\left(E^{0} \rightarrow E^{1}\right) \simeq \frac{\prod_{n=d_{p}}^{\infty} K_{p}^{n}}{\Theta_{n=d_{p}}^{\infty} K_{p}^{n}}$.

Let $M_{p}=\frac{\prod_{n=d_{d}}^{\infty} K_{p}^{n}}{\oplus_{n=d_{p}}^{\infty} K_{p}^{n}}$. Since $M_{p}$ is an $R_{p}$-module which is injective as an $R$-module it follows (by Lemma 5) that $M_{p}$ is an injective $R_{p}$-module.

$K_{p}^{n}$ is not injective for any $n \geq 0$. So for each $n \geq 0$ there is $f_{n} \in$ $\operatorname{Hom}_{R_{p}}\left(k(p), K_{p}^{n}\right)$ that can not be extended to a homomorphism $E(k(p)) \rightarrow$ $K_{p}^{n}$.

Let $f_{n}\left(1+p R_{p}\right)=x_{p}^{n}$.

Let $f: k(p) \rightarrow M_{p}$ be defined by $f\left(1+p R_{p}\right)=\left(x_{p}^{n}\right)_{n \geq d_{p}}+\oplus_{n=d_{p}}^{\infty} K_{p}^{n}$. $f$.

Since $M_{p}$ is injective there is $F \in \operatorname{Hom}_{R_{p}}\left(E(k(p)), M_{p}\right)$ such that $\left.F\right|_{k(p)}=$

The sequence $0 \rightarrow \oplus_{n=d_{p}}^{\infty} K_{p}^{n} \rightarrow \Pi_{n=d_{p}}^{\infty} K_{p}^{n} \stackrel{\theta}{\longrightarrow} M_{p} \rightarrow 0$ is exact. Therefore we have the long exact sequence $0 \rightarrow \operatorname{Hom}\left(E(k(p)), \oplus_{n=d_{p}}^{\infty} K_{p}^{n}\right) \rightarrow$ $\operatorname{Hom}\left(E(k(p)), \Pi_{n=d_{p}}^{\infty} K_{p}^{n}\right) \longrightarrow \operatorname{Hom}\left(E(k(p)), M_{p}\right) \longrightarrow \operatorname{Ext}^{1}(E(k(p))$, $\left.\oplus_{n=d_{p}}^{\infty} K_{p}^{n}\right)=0\left(\right.$ since $\oplus_{n=d_{p}}^{\infty} K_{p}^{n}$ is Gorenstein injective $)$.

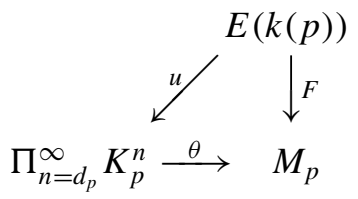


So there exists $u \in \operatorname{Hom}\left(E(k(p)), \Pi_{n=d_{p}}^{\infty} K_{p}^{n}\right)$ such that $\theta \circ u=F$, with $\theta: \Pi_{n=d_{p}}^{\infty} K_{p}^{n} \rightarrow M_{p}, \theta\left(\left(z^{n}\right)_{n \geq d_{p}}\right)=\left(z^{n}\right)_{n \geq d_{p}}+\oplus_{n=d_{p}}^{\infty} K_{p}^{n}$.

Let $u\left(1+p R_{p}\right)=\left(y_{p}^{n}\right)_{n \geq d_{p}}$.

Then $F\left(1+p R_{p}\right)=\theta\left(\left(y_{p}^{n}\right)_{n \geq d_{p}}\right)=\left(y_{p}^{n}\right)_{n \geq d_{p}}+\oplus_{n=d_{p}}^{\infty} K_{p}^{n}$.

But $\left.F\right|_{k(p)}=f$, so $F\left(1+p R_{p}\right)=f\left(1+p R_{p}\right)=\left(x_{p}^{n}\right)_{n \geq d_{p}}+\oplus_{n=d_{p}}^{\infty} K_{p}^{n}$.

Hence $x_{p}^{n}=y_{p}^{n}$ for all but finitely many $n \geq d_{p}$.

If $n \geq d_{p}$ is such that $x_{p}^{n}=y_{p}^{n}$ then $\pi_{n} \circ u \in \operatorname{Hom}_{R_{p}}\left(E(k(p)), K_{p}^{n}\right)$ and $\left.\pi_{n} \circ u\right|_{k(p)}=f_{n}$ (with $\left.\pi_{n}: \Pi_{j=d_{p}}^{\infty} K_{p}^{j} \rightarrow K_{p}^{n}, \pi_{n}\left(\left(z^{j}\right)_{j}\right)=z^{n}\right)$. So $f_{n} \in \operatorname{Hom}\left(k(p), K_{p}^{n}\right)$ can be extended to a homomorphism $E(k(p)) \rightarrow K_{p}^{n}$. Contradiction.

So inj $\operatorname{dim}_{R_{p}} k(p)<\infty \Leftrightarrow$ proj $\operatorname{dim}_{R_{p}} k(p)<\infty \Leftrightarrow \operatorname{gl}$ dim $R_{p}<\infty$.

Since $R_{p}$ is a local ring and gl.dim $R_{p}<\infty$ it follows that gl.dim $R_{p}=$ Krull $\operatorname{dim} R_{p}$ ([11], Theorem 4.4.16). By [3], Corollary 3.4, Krull $\operatorname{dim} R_{p}=$ inj $\operatorname{dim}_{R_{p}} R_{p}$. So

$$
\text { gl.dim } R_{p}=\operatorname{inj} \operatorname{dim}_{R_{p}} R_{p}, \forall p \in \operatorname{Spec} R .
$$

Let $\operatorname{inj} \operatorname{dim}_{R} R=n<\infty$.

By [3], Corollary 2.3, we have

$$
\operatorname{inj} \operatorname{dim}_{R_{p}} R_{p} \leq \operatorname{inj} \operatorname{dim}_{R} R=n, \forall p \in \operatorname{Spec} R .
$$

By (3) and (4), gl.dim $R_{p} \leq n, \forall p \in \operatorname{Spec} R$.

By [7], Theorem 9.52, gl.dim $R=\sup$ gl.dim $R_{m}$ when $m$ ranges over all maximal ideals in $R$.

Hence gl.dim $R \leq n$.

(2) $\Rightarrow$ (1) Since gl.dim $R<\infty$ it follows that a complex $J$ is DG-injective if and only if each $J^{n}$ is an injective module.

Let $\left(J_{i}\right)_{i \in I}$ be a family of DG-injective complexes.

Since $R$ is noetherian and $J_{i}^{n}$ is injective $\forall i \in I$ it follows that $\oplus_{i \in I} J_{i}^{n}$ is injective. So $\bigoplus_{i \in I} J_{i}$ is a DG-injective complex.

THEOREM 4. If $R$ is a commutative Gorenstein ring then every direct sum of exact covers is an exact cover if and only if $\operatorname{gl} . \operatorname{dim} R<\infty$.

Proof. " $\Rightarrow$ " Let $\left(J_{i}\right)_{i \in i}$ be a family of DG-injective complexes.

By Proposition $2 J_{i}=D_{i} \oplus K_{i}$ with $D_{i}$ injective and $K_{i}$ minimal DGinjective.

By Lemma $1, \mathrm{Id}_{i}: K_{i} \rightarrow K_{i}$ gives the exact sequence $0 \rightarrow K_{i} \rightarrow$ $M\left(\operatorname{Id}_{i}\right) \stackrel{\psi_{i}}{\longrightarrow} K_{i}[1] \rightarrow 0$ with $M\left(\operatorname{Id}_{i}\right) \stackrel{\text { not }}{=} E_{i}$ ("not" is for notation) an injective complex and with $E_{i} \rightarrow K_{i}[1]$ an exact cover.

By hypothesis $\oplus_{i \in I} E_{i} \rightarrow \bigoplus_{i \in I} K_{i}[1]$ is an exact cover. 
So $\oplus_{i \in I} K_{i}=\operatorname{Ker}\left(\oplus_{i \in I} E_{i} \rightarrow \oplus_{i \in I} K_{i}[1]\right)$ is minimal DG-injective. We have $\oplus_{i \in I} J_{i}=\left(\oplus_{i \in I} D_{i}\right) \oplus\left(\oplus_{i \in I} K_{i}\right)$ with $\oplus_{i \in I} D_{i}$ injective (hence DG-injective) and $\oplus_{i \in I} K_{i}$ DG-injective. Since the class of DG-injective complexes is closed under taking finite direct sums ([6], pp. 27) it follows that $\oplus_{i \in I} J_{i}$ is DGinjective.

Since $R$ is commutative Gorenstein and every direct sum of DG-injective complexes is DG-injective, it follows that gl.dim $R<\infty$ (by Theorem 3).

" $\Leftarrow$ " Let $E_{i} \stackrel{\psi_{i}}{\longrightarrow} X_{i}$ be an exact cover, for any $i \in I$.

Then $T_{i}=\operatorname{Ker} \psi_{i}$ is a minimal DG-injective complex (Theorem 1).

Each sequence $0 \rightarrow T_{i} \rightarrow E_{i} \rightarrow X_{i} \rightarrow 0$ is exact, so the sequence $0 \rightarrow \oplus_{i \in I} T_{i} \rightarrow \oplus_{i \in I} E_{i} \rightarrow \oplus_{i \in I} X_{i} \rightarrow 0$ is exact.

Since gl.dim $R<\infty$ it follows that $\oplus_{i \in I} T_{i}$ is DG-injective.

$T_{i}=\ldots \rightarrow T_{i}^{n-1} \stackrel{f_{i}^{n-1}}{\longrightarrow} T_{i}^{n} \stackrel{f_{i}^{n}}{\longrightarrow} T_{1}^{n+1} \rightarrow \ldots$

For each $i \in I, \operatorname{Ker} f_{i}^{n}$ is essential in $T_{i}^{n}$, so $\oplus_{i \in I} \operatorname{Ker} f_{i}^{n}$ is essential in $\oplus_{i \in I} T_{i}^{n}, \forall n \in \mathbf{Z}$ ([5], Exercise 11, pp. 75).

Hence $\oplus_{i \in I} T_{i}$ is minimal DG-injective.

Since $\oplus_{i \in I} E_{i} \rightarrow \oplus_{i \in I} X_{i}$ is surjective, $\oplus_{i \in I} E_{i}$ is exact and $\operatorname{Ker}\left(\oplus_{i \in I} E_{i} \rightarrow\right.$ $\left.\oplus_{i \in I} X_{i}\right)$ is minimal DG-injective it follows that $\oplus_{i \in I} E_{i} \rightarrow \oplus_{i \in I} X_{i}$ is an exact cover (by Theorem 1).

\section{Minimal projective resolutions of finitely generated modules over local noetherian rings}

We consider a complete commutative local noetherian ring $R$ with the property that every direct sum of DG-injective complexes of $R$-modules is DG-injective.

$R$ is local, so every finitely generated $R$-module has a minimal projective resolution. Let $\ldots \rightarrow R^{\beta_{1}} \stackrel{f_{1}}{\longrightarrow} R^{\beta_{0}} \stackrel{f_{0}}{\longrightarrow} M \rightarrow 0$ be a minimal projective resolution of a finitely generated $R$-module $M$. We show (Theorem 5) that for each $l \geq 1$ there is $\bar{n} \geq 1$ such that the entries of the matrix that represents $f_{n}$ are all in $m^{l}$, for any $n \geq \bar{n}$.

Theorem 6 proves that the result is true for any commutative local noetherian ring $R$ with the property that the direct sum of DG-injective complexes is DGinjective.

Our first result in this section is the following.

Lemma 6. Let $(R, m, k)$ be a commutative local noetherian ring. For each $n \geq 1$ let $E_{n}=\left\{x \mid x \in E(k), m^{n} x=0\right\}$.

If $K \leq E(k)^{s}$ then $E_{n}^{s} \subset K$ if and only if (1) $k^{s} \subset K$ and (2) any linear map $k \rightarrow K$ has an extension $E_{n} \rightarrow K$. 
Proof. Since $k^{s} \subset E(k)^{s}$ is an injective envelope over $R$ and $m^{n} k^{s}=0$ it follows that the injective envelope of $k^{s}$ over $R / m^{n}$ is $\left\{x \in E(k)^{s} \mid m^{n} x=\right.$ $0\}=E_{n}^{s}$.

" $\Leftarrow$ " Let $f_{1}, \ldots, f_{s} \in \operatorname{Hom}(k, K), f_{1}(x)=(x, 0, \ldots, 0), \ldots, f_{s}(x)=$ $(0,0, \ldots, x)$. By hypothesis there is $\sigma_{j} \in \operatorname{Hom}_{R}\left(E_{n}, K\right)$ that extends $f_{j}, 1 \leq$ $j \leq s$. Let $\sigma: E_{n}^{s} \rightarrow K, \sigma\left(y_{1}, \ldots, y_{s}\right)=\sigma_{1}\left(y_{1}\right)+\cdots+\sigma_{s}\left(y_{s}\right)$.

If $y=\left(y_{1}, \ldots, y_{s}\right) \in k^{s} \cap \operatorname{Ker} \sigma$ then $\left(y_{1}, \ldots, y_{s}\right)=f_{1}\left(y_{1}\right)+\ldots+f_{s}\left(y_{s}\right)=$ $\sigma_{1}\left(y_{1}\right)+\cdots+\sigma_{s}\left(y_{s}\right)=0$. Since $k^{s}$ is essential in $E_{n}^{s}$ and $k^{s} \cap \operatorname{Ker} \sigma=0$ it follows that $\operatorname{Ker} \sigma=0$.

So $E_{n}^{s} \simeq \sigma\left(E_{n}^{s}\right) \subset K$. Therefore $k^{s}=\sigma\left(k^{s}\right) \subset \sigma\left(E_{n}^{s}\right)$ is an injective envelope over $R / m^{n}$.

We have $r \sigma\left(y_{1}, \ldots, y_{s}\right)=r \sigma_{1}\left(y_{1}\right)+\cdots+r \sigma_{s}\left(y_{s}\right)=\sigma_{1}\left(r y_{1}\right)+\cdots+$ $\sigma_{s}\left(r y_{s}\right)=0$, for any $r \in m^{n}$, for any $\left(y_{1}, \ldots, y_{s}\right) \in E_{n}^{s}$. So $\sigma\left(E_{n}^{s}\right) \subset\{x \in$ $\left.K \mid m^{n} x=0\right\} \subset\left\{x \in E(k)^{s} \mid m^{n} x=0\right\}=E_{n}^{s}$.

Both $\sigma\left(E_{n}^{s}\right)$ and $E_{n}^{s}$ are injective envelopes of $k^{s}$, and $\sigma\left(E_{n}^{s}\right) \subset E_{n}^{s}$. So $\sigma\left(E_{n}^{s}\right)=E_{n}^{s}$ as $R / m^{n}$-modules. Since their structure of $R / m^{n}$-modules is given by $\left(r+m^{n}\right) x=r \cdot x$, for any $r+m^{n} \in R / m^{n}$, it follows that $\sigma\left(E_{n}^{s}\right)=E_{n}^{s}$ as $R$-modules. So $E_{n}^{s} \subset K$.

" $\Rightarrow$ " We have $E_{n}^{s} \subset K$, so $k^{s} \subset E_{n}^{s} \subset K$.

Let $f \in \operatorname{Hom}_{R}(k, K), f \neq 0$. Since $k$ is simple, $f$ is injective, so $f(k) \simeq k$. Since $f(k) \cap k^{s} \leq f(k)$ and $f(k)$ is simple it follows that $f(k) \cap k^{s}=0$ or $f(k) \cap k^{s}=f(k) . k^{s}$ is essential in $E(k)^{s}$, so $f(k) \cap k^{s}=0$ would imply $f(k)=0 \Leftrightarrow k=0$. False. Hence $f(k) \cap k^{s}=f(k) \Leftrightarrow f(k) \subset k^{s}$.

$f$ is an $R$-homomorphism, so it is also an $R / m^{n}$-homomorphism.

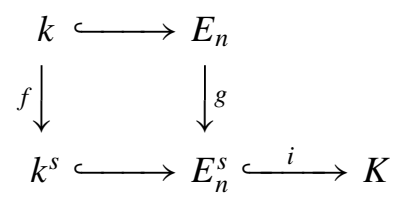

Since $k \subset E_{n}$ and $k^{s} \subset E_{n}^{s}$ are injective envelopes over $R / m^{n}$, there is $g \in \operatorname{Hom}_{R / m^{n}}\left(E_{n}, E_{n}^{s}\right)$ that extends $f$.

For any $r \in R$ we have $g(r \cdot x)=g\left(\left(r+m^{n}\right) x\right)=\left(r+m^{n}\right) g(x)=r \cdot g(x)$. So $g \in \operatorname{Hom}_{R}\left(E_{n}, E_{n}^{s}\right)$. The homomorphism $i \circ g$ extends $f$.

We use Lemma 6 to prove the following result.

Proposition 4. Let $(R, m, k)$ be a commutative local noetherian ring such that every direct sum of DG-injective complexes of $R$-modules is DG-injective. Let $l \geq 1$. If $0 \rightarrow A \rightarrow E^{0} \rightarrow E^{1} \rightarrow \ldots$ is a minimal injective resolution of an artinian $R$-module $A$, then there is $n_{0} \geq 0$ such that $\left\{x \in E^{n} \mid m^{l} x=\right.$ $0\} \subset \operatorname{Ker}\left(E^{n} \rightarrow E^{n+1}\right)$, for all $n \geq n_{0}$. 
Proof. Since $A$ is an artinian module, we have $E^{n}=E(k)^{\beta_{n}}$, for some $\beta_{n} \geq 1$ (Lemma 3).

Let $E_{l}=\left\{x \in E(k) \mid m^{l} x=0\right\}$.

Since $R / m^{l}$ has finite length, it follows that $\operatorname{Hom}\left(R / m^{l}, E(k)\right)$ has finite length ([5], Theorem 3.4.1). So $E_{l} \simeq \operatorname{Hom}\left(R / m^{l}, E(k)\right)$ is of finite length. By [5], Theorem 2.3.17, $E_{l}$ is noetherian, hence finitely generated.

Let $K_{n}=\operatorname{Ker}\left(E^{n} \rightarrow E^{n+1}\right)$ for any $n \geq 0$.

We prove that there is $d_{0} \geq 0$ such that any linear map $k \rightarrow K_{d}$ can be extended to a linear map $E_{l} \rightarrow K_{d}$, for any $d \geq d_{0}$.

Suppose no $d_{0} \geq 0$ has this property. Then for any $j \geq 0$ there is $d_{j} \geq j$ and $f_{d_{j}} \in \operatorname{Hom}\left(k, K_{d_{j}}\right)$ that cannot be extended to $E_{l} \rightarrow K_{d_{j}}$.

Each of the complexes

$$
\begin{aligned}
& J_{d_{0}}=\ldots \rightarrow 0 \rightarrow 0 \rightarrow 0 \rightarrow E^{d_{0}} \stackrel{f_{d_{0}}}{\rightarrow} E^{d_{0}+1} \rightarrow E^{d_{0}+2} \rightarrow \ldots \\
& J_{d_{1}}=\ldots \rightarrow 0 \rightarrow 0 \rightarrow E^{d_{1}-1} \rightarrow E^{d_{1}} \stackrel{f_{d_{1}}}{\rightarrow} E^{d_{1}+1} \rightarrow E^{d_{1}+2} \rightarrow \ldots \\
& J_{d_{2}}=\ldots \rightarrow 0 \rightarrow E^{d_{2}-2} \rightarrow E^{d_{2}-1} \rightarrow E^{d_{2}} \stackrel{f_{d_{2}}}{\rightarrow} E^{d_{2}+1} \rightarrow E^{d_{2}+2} \rightarrow \ldots
\end{aligned}
$$

is DG-injective so, by hypothesis, $\oplus_{i=0}^{\infty} J_{d_{i}}$ is DG-injective.

If $0 \rightarrow \oplus_{i=0}^{\infty} J_{d_{i}} \stackrel{\psi}{\longrightarrow} \Pi_{i=0}^{\infty} J_{d_{i}} \stackrel{P}{\longrightarrow} F \rightarrow 0$ is exact then $F$ is an exact complex (since $\psi$ is a quasi isomorphism). Both $\oplus_{i=0}^{\infty} J_{d_{i}}$ and $\prod_{i=0}^{\infty} J_{d_{i}}$ are DGinjective, so $F$ is a DG-injective complex. Thus $F$ is an injective complex ([6], Proposition 3.7) and therefore $\operatorname{Ker}\left(F^{n} \rightarrow F^{n+1}\right)$ is an injective $R$-module for any $n \in \mathbf{Z}$. In particular,

$$
\operatorname{Ker}\left(F^{0} \rightarrow F^{1}\right) \simeq \frac{\prod_{j=0}^{\infty} \operatorname{Ker} f_{d_{j}}}{\oplus_{j=0}^{\infty} \operatorname{Ker} f_{d_{j}}}=\frac{\prod_{j=0}^{\infty} K_{d_{j}}}{\oplus_{j=0}^{\infty} K_{d_{j}}}
$$

is injective.

Let $M=\frac{\prod_{j=0}^{\infty} K_{d_{j}}}{\oplus_{j=0}^{\infty} K_{d_{j}}}$.

Let $f_{d_{j}}(1+m)=x_{d_{j}}$ and let $f: k \rightarrow M$ be defined by $f(1+m)=$ $\left(x_{d_{j}}\right)_{j \geq 0}+\oplus_{j=0}^{\infty} K_{d_{j}}$.

Since $M$ is injective there is $g \in \operatorname{Hom}\left(E_{l}, M\right)$ that extends $f$. Since $E_{l}$ is finitely generated and $\oplus_{j=0}^{\infty} K_{d_{j}}$ is a pure submodule of $\Pi_{j=0}^{\infty} K_{d_{j}}$, it follows that the sequence $0 \rightarrow \operatorname{Hom}\left(E_{l}, \oplus_{j=0}^{\infty} K_{d_{j}}\right) \rightarrow \operatorname{Hom}\left(E_{l}, \Pi_{j=0}^{\infty} K_{d_{j}}\right) \rightarrow$ $\operatorname{Hom}\left(E_{l}, M\right) \rightarrow 0$ is exact. So there is $u \in \operatorname{Hom}\left(E_{l}, \Pi_{j=0}^{\infty} K_{d_{j}}\right)$ such that $g=\theta \circ u$ (with $\left.\theta\left(\left(z_{d_{j}}\right)\right)=\left(z_{d_{j}}\right)_{j \geq 0}+\oplus_{j=0}^{\infty} K_{d_{j}}\right)$.

If $u(1+m)=\left(y_{d_{j}}\right)_{j \geq 0}$ then $g(1+m)=\theta(u(1+m))=\theta\left(\left(y_{d_{j}}\right)_{j}\right)=$ $\left(y_{d_{j}}\right)_{j \geq 0}+\oplus_{j=0}^{\infty} K_{d_{j}}$. 
Since $\left.g\right|_{k}=f$ we have $g(1+m)=f(1+m)=\left(x_{d_{j}}\right)_{j \geq 0}+\oplus_{j=0}^{\infty} K_{d_{j}}$. So $x_{d_{j}}=y_{d_{j}}$ for all but finitely many $j \geq 0$.

Let $j \geq 0$ be such that $x_{d_{j}}=y_{d_{j}}$ and let $\pi_{d_{j}}: \Pi_{l=0}^{\infty} K_{d_{l}} \rightarrow K_{d_{j}}, \pi_{d_{j}}\left(\left(z_{d_{l}}\right)_{l}\right)=$ $z_{d_{j}}$. We have $\pi_{d_{j}} \circ u(1+m)=\pi_{d_{j}}\left(\left(y_{d_{l}}\right)_{l}\right)=y_{d_{j}}=x_{d_{j}}=f(1+m)$.

Hence $\pi_{d_{j}} \circ u$ extends $f_{d_{j}}$. Contradiction.

So there is $d_{0} \geq 0$ such that for any $d \geq d_{0}$, any linear map $k \rightarrow K_{d}$ can be extended to $E_{l} \rightarrow K_{d}$.

By [5], Corollary 3.1.21, we have Soc $K_{d}=\operatorname{Soc} E\left(K_{d}\right)=\operatorname{Soc} E(k)^{\beta_{d}}=$ $k^{\beta_{d}}$. So $k^{\beta_{d}} \subset K_{d}$ for all $d \geq 0$.

Since $k^{\beta_{d}} \subset K_{d} \subset E(k)^{\beta_{d}}$ and any linear map $k \rightarrow K_{d}$ can be extended to $E_{l} \rightarrow K_{d}$ it follows (Lemma 6) that $E_{l}^{\beta_{d}} \subset K_{d} \Leftrightarrow\left\{x \in E(k)^{\beta_{d}} \mid m^{l} x=0\right\} \subset$ $K_{d}$. So $\left\{x \in E^{d} \mid m^{l} x=0\right\} \subset K_{d}$, for any $d \geq d_{0}$.

We can prove now the following.

THeOREM 5. Let $(R, m, k)$ be a complete commutative local noetherian ring with the property that every direct sum of DG-injective complexes of $R$ modules is DG-injective. Let $M$ be a finitely generated $R$-module. If $\ldots \rightarrow$ $R^{\beta_{1}} \stackrel{f_{1}}{\longrightarrow} R^{\beta_{0}} \stackrel{f_{0}}{\longrightarrow} M \rightarrow 0$ is a minimal projective resolution of $M$ then for each $l \geq 1$ there is $\bar{n} \geq 1$ such that the entries of the matrix that represents $f_{n}$ are all in $m^{l}$, for any $n \geq \bar{n}$.

Proof. Since $E(k)$ is injective and the complex $\ldots \rightarrow R^{\beta_{1}} \stackrel{f_{1}}{\longrightarrow} R^{\beta_{0}} \stackrel{f_{0}}{\longrightarrow}$ $M \rightarrow 0$ is exact, it follows that $0 \rightarrow M^{v} \rightarrow\left(R^{\beta_{0}}\right)^{v} \rightarrow\left(R^{\beta_{1}}\right)^{v} \rightarrow \ldots$ is an exact complex.

For each $n \geq 0,\left(R^{\beta_{n}}\right)^{v}=\operatorname{Hom}\left(R^{\beta_{n}}, E(k)\right) \simeq \operatorname{Hom}(R, E(k))^{\beta_{n}} \simeq E(k)^{\beta_{n}}$ is an injective $R$-module. So $0 \rightarrow M^{v} \stackrel{f_{0}^{v}}{\longrightarrow}\left(R^{\beta_{0}}\right)^{v} \stackrel{f_{1}^{v}}{\longrightarrow}\left(R^{\beta_{1}}\right)^{v} \rightarrow \ldots$ is an injective resolution of $M^{v}$.

If $u \in \operatorname{Hom}\left(\left(R^{\beta_{0}}\right)^{v},\left(R^{\beta_{0}}\right)^{v}\right)$ is such that $u \circ f_{0}^{v}=f_{0}^{v}$, then $f_{0}^{v v} \circ u^{v}=f_{0}^{v v}$.

Since $R$ is complete and ${ }_{R} M$ is finitely generated it follows that $M \simeq M^{v v}$ ([5], Lemma 3.4.6). Similarly $R^{\beta_{0}} \simeq\left(R^{\beta_{0}}\right)^{v v}$.

So $f_{0}^{v v}:\left(R^{\beta_{0}}\right)^{v v} \rightarrow M^{v v}$ is a projective cover. Thus $f_{0}^{v v} \circ u^{v}=f_{0}^{v v}$ implies $u^{v} \in \operatorname{Aut}\left(R^{\beta_{0}}\right)^{v \nu}$. Then $u^{\nu v} \in \operatorname{Aut}\left(R^{\beta_{0}}\right)^{v v v}$.

The diagram

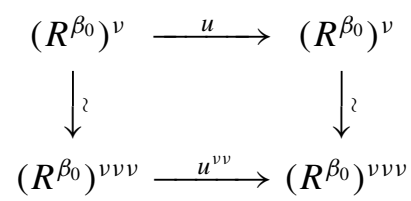

is commutative. So $u^{\nu v} \in \operatorname{Aut}\left(R^{\beta_{0}}\right)^{v v v}$ implies that $u \in \operatorname{Aut}\left(R^{\beta_{0}}\right)^{\nu}$. 
Hence $M^{v} \rightarrow\left(R^{\beta_{0}}\right)^{v}$ is an injective envelope of $M^{v}$. Similarly, $\left(\operatorname{Im} f_{n}\right)^{v}$ $\stackrel{f_{n+1}^{v}}{\longrightarrow}\left(R^{\beta_{n+1}}\right)^{v}$ is an injective envelope, for any $n \geq 1$. So $0 \rightarrow M^{v} \stackrel{f_{0}^{v}}{\longrightarrow}$ $\left(R^{\beta_{0}}\right)^{v} \stackrel{f_{1}^{v}}{\longrightarrow}\left(R^{\beta_{1}}\right)^{v} \stackrel{f_{2}^{v}}{\longrightarrow} \ldots$ is a minimal injective resolution of the $R$-module $M^{v}$.

The ring $R$ is complete and ${ }_{R} M$ is noetherian, so $M^{v}$ is an artinian $R$-module ([5], Corollary 3.4.4).

Let $l \geq 1$. Let $E_{l}=\left\{x \in E(k) \mid m^{l} x=0\right\}$.

By Proposition 4 there is $n_{0} \geq 1$ such that $\left\{x \in\left(R^{\beta_{n}}\right)^{v} \mid m^{l} x=0\right\} \subset$ Ker $f_{n+1}^{v}$, for any $n \geq n_{0}$.

Let $n \geq n_{0}$.

Let $\theta \in\left(R^{\beta_{n}}\right)^{v}$ be defined by $\theta\left(e_{1}^{\beta_{n}}\right)=y$, for some $y \in E_{l}$, and $\theta\left(e_{j}^{\beta_{n}}\right)=0$, $2 \leq j \leq \beta_{n}$, where

$$
e_{1}^{\beta_{n}}=\left(\begin{array}{c}
1 \\
0 \\
\vdots \\
0
\end{array}\right), \quad e_{2}^{\beta_{n}}=\left(\begin{array}{c}
0 \\
1 \\
\vdots \\
0
\end{array}\right), \quad \ldots, \quad e_{\beta_{n}}^{\beta_{n}}=\left(\begin{array}{c}
0 \\
0 \\
\vdots \\
1
\end{array}\right) .
$$

Since $m^{l} \theta=0$, it follows that $f_{n+1}^{v}(\theta)=0$, which is equivalent to $\theta \circ f_{n+1}=0$.

$$
f_{n+1}: R^{\beta_{n+1}} \rightarrow R^{\beta_{n}}, \quad f_{n+1}(x)=\left(\begin{array}{cccc}
a_{11} & a_{21} & \ldots & a_{\beta_{n+1} 1} \\
a_{12} & a_{22} & \ldots & a_{\beta_{n+1} 2} \\
\vdots & \vdots & & \vdots \\
a_{1 \beta_{n}} & a_{2 \beta_{n}} & \ldots & a_{\beta_{n+1} \beta_{n}}
\end{array}\right)\left(\begin{array}{c}
x_{1} \\
x_{2} \\
\vdots \\
x_{\beta_{n+1}}
\end{array}\right)
$$

for any

$$
\left(\begin{array}{c}
x_{1} \\
x_{2} \\
\vdots \\
x_{\beta_{n+1}}
\end{array}\right) \in R^{\beta_{n+1}}, \quad \text { where } \quad f_{n+1}\left(e_{j}^{\beta_{n+1}}\right)=\left(\begin{array}{c}
a_{j 1} \\
a_{j 2} \\
\vdots \\
a_{j \beta_{n}}
\end{array}\right), \quad 1 \leq j \leq \beta_{n+1} .
$$

$\theta \circ f_{n+1}\left(e_{1}^{\beta_{n+1}}\right)=0 \Leftrightarrow \theta\left(a_{11} e_{1}^{\beta_{n}}+a_{12} e_{2}^{\beta_{n}}+\cdots+a_{1 \beta_{n}} e_{\beta_{n}}^{\beta_{n}}\right)=0 \Leftrightarrow a_{11} \theta\left(e_{1}^{\beta_{n}}\right)+$ $\cdots+a_{1 \beta_{n}} \theta\left(e_{\beta_{n}}^{\beta_{n}}\right)=0 \Leftrightarrow a_{11} y=0$. Since $a_{11} \in$ Ann $y$, for any $y \in E_{l}$ we have $a_{11} \in$ Ann $E_{l}=m^{l}$. Similarly $a_{i j} \in m^{l}$ for any $i \in\left\{1, \ldots, \beta_{n+1}\right\}$, and for any $j \in\left\{1, \ldots, \beta_{n}\right\}$, for any $n \geq n_{0}$.

Theorem 6 shows that we can drop the completeness as part of the hypothesis of Theorem 5.

The proof of Theorem 6 uses the following. 
Lemma 7. Let $(R, m, k)$ be a commutative local noetherian ring and let $\hat{R}$ be the $m$-adic completion of $R$. If $E$ is an $\hat{R}$-module, then $E$ is an injective $\hat{R}$-module if and only if $E$ is an injective $R$-module.

Proof. " $\Rightarrow$ " Since $\hat{R}$ is a flat $R$-module and $E$ is an injective $\hat{R}$-module, it follows ([5], Theorem 3.2.9) that $\operatorname{Hom}_{\hat{R}}(\hat{R}, E)$ is an injective $R$-module. $\operatorname{Hom}_{\hat{R}}(\hat{R}, E) \simeq E$ as $\hat{R}$-modules, so also as $R$-modules (when restricting the scalars). Thus $E$ is an injective $R$-module.

" $\Leftarrow$ " Let $J$ be an ideal of $\hat{R}$. Then $J=\hat{R} \otimes_{R} I$ for some ideal $I$ of $R$. By [5], Theorem 2.1.10, $\operatorname{Hom}_{\hat{R}}\left(\hat{R} \otimes_{R} I, E\right) \simeq \operatorname{Hom}_{R}\left(I, \operatorname{Hom}_{\hat{R}}(\hat{R}, E)\right) \simeq$ $\operatorname{Hom}_{R}(I, E)$. Similarly, $\operatorname{Hom}_{\hat{R}}\left(\hat{R} \otimes_{R} R, E\right) \simeq \operatorname{Hom}_{R}(R, E)$.

Since $0 \rightarrow I \rightarrow R$ is exact and $E$ is injective it follows that the sequence $\operatorname{Hom}_{R}(R, E) \rightarrow \operatorname{Hom}_{R}(I, E) \rightarrow 0$ is exact $\Leftrightarrow \operatorname{Hom}_{\hat{R}}(\hat{R}, E) \rightarrow$ $\operatorname{Hom}_{\hat{R}}(J, E) \rightarrow 0$ is exact.

So $E$ is an injective $\hat{R}$-module.

THEOREM 6. Let $(R, m, k)$ be a commutative local noetherian ring with the property that every direct sum of DG-injective complexes of $R$-modules is DG-injective. Let $M$ be a finitely generated $R$-module. If $\ldots \rightarrow R^{\beta_{1}} \stackrel{f_{1}}{\longrightarrow}$ $R^{\beta_{0}} \stackrel{f_{0}}{\longrightarrow} M \rightarrow 0$ is a minimal projective resolution of $M$ then for each $l \geq 1$ there is $\bar{n} \geq 1$ such that the entries of the matrix that represents $f_{n}$ are all in $m^{l}$ for any $n \geq \bar{n}$.

Proof. $\hat{R}$ is a commutative local noetherian ring with maximal ideal $m \hat{R}$ and residue field $\hat{R} / m \hat{R} \simeq(\widehat{R / m})=k$ ([5], pp. 66).

Since the complex $\ldots \rightarrow R^{\beta_{1}} \stackrel{f_{1}}{\longrightarrow} R^{\beta_{0}} \stackrel{f_{0}}{\longrightarrow} M \rightarrow 0$ is exact and $\hat{R}$ is a flat $R$-module ([5], Theorem 2.5.18), it follows that $\ldots \rightarrow \hat{R} \otimes_{R} R^{\beta_{1}} \rightarrow$ $\hat{R} \otimes_{R} R^{\beta_{0}} \rightarrow \hat{R} \otimes_{R} M \rightarrow 0$ is an exact complex.

By [5], Theorem 2.5.14, $\hat{R} \otimes_{R} M \simeq \hat{M}$.

We have $\hat{R} \otimes_{R} R^{n} \simeq \hat{R} \otimes_{R}(R \oplus \ldots \oplus R) \simeq \hat{R} \otimes_{R} R \oplus \ldots \oplus \hat{R} \otimes_{R} R \simeq \hat{R}^{n}$, for any $n \geq 1$.

So $\ldots \rightarrow \hat{R}^{\beta_{1}} \stackrel{\hat{f}_{1}}{\longrightarrow} \hat{R}^{\beta_{0}} \stackrel{\hat{f}_{0}}{\longrightarrow} \hat{M} \rightarrow 0$ is a free resolution of $\hat{R^{M}}$.

$R^{\beta_{0}} \rightarrow M$ is a projective cover, so $M / m M \simeq(R / m)^{\beta_{0}}$.

Since $M$ is finitely generated we have $\widehat{m M}=m \hat{M}$. So $\hat{M} / m \hat{M}=\hat{M} / \widehat{m M}$ $\simeq M \widehat{/ m} M \simeq(\widehat{R / m})^{\beta_{0}} \simeq(\hat{R} / \hat{m})^{\beta_{0}}$. Hence $\hat{R}^{\beta_{0}} \stackrel{\hat{f}_{0}}{\longrightarrow} \hat{M}$ is a projective cover. Similarly, $\hat{R}^{\beta_{n}} \stackrel{\hat{f}_{n}}{\longrightarrow} \operatorname{Im} \hat{f}_{n}$ is a projective cover, for any $n \geq 1$. So $\ldots \rightarrow$ $\hat{R}^{\beta_{1}} \stackrel{\hat{f}_{1}}{\longrightarrow} \hat{R}^{\beta_{0}} \stackrel{\hat{f}_{0}}{\longrightarrow} \hat{M} \rightarrow 0$ is a minimal projective resolution of $\hat{M}$.

By [5], Theorem 3.4.1, $E(k) \simeq E_{\hat{R}}(\hat{R} / \hat{m})$ as an $\hat{R}$-module, so the complex $0 \rightarrow \hat{M}^{v} \rightarrow\left(\hat{R}^{\beta_{0}}\right)^{v} \rightarrow\left(\hat{R}^{\beta_{1}}\right)^{v} \rightarrow \ldots$ is exact. The same argument used in 
the proof of Theorem 5 shows that $0 \rightarrow \hat{M} \stackrel{\hat{f}_{0}^{v}}{\longrightarrow}\left(\hat{R}^{\beta_{0}}\right)^{v} \stackrel{\hat{f}_{1}^{v}}{\longrightarrow}\left(\hat{R}^{\beta_{1}}\right)^{v} \rightarrow \ldots$ is a minimal injective resolution of $\hat{R}_{\hat{M}} \hat{M}^{\nu}$. Since $\hat{R}$ is complete and ${ }_{R} \hat{M}$ is noetherian it follows that $\hat{M}^{v}$ is an artinian $\hat{R}$-module ([5], Corollary 3.4.4).

Let $E^{n}=\left(\hat{R}^{\beta_{n}}\right)^{v}$ and let $K_{n}=\operatorname{Ker}\left(E^{n} \rightarrow E^{n+1}\right)$ for any $n \geq 0$. Let $E_{l}=\left\{x \in E(k) \mid \hat{m}^{l} x=0\right\}$. Since $E_{l} \simeq \operatorname{Hom}_{\hat{R}}\left(\hat{R} / \hat{m}^{l}, E(k)\right)$ and $\hat{R} / \hat{m}^{l}$ has finite length it follows that $E_{l}$ has finite length. By [5], Theorem 2.3.17, $E_{l}$ is finitely generated.

We prove that there is $d_{0} \geq 0$ such that any linear map $k \rightarrow K_{d}$ can be extended to a linear map $E_{l} \rightarrow K_{d}$, for any $d \geq d_{0}$.

Suppose no $d_{0} \geq 0$ has this property. Then for any $j \geq 0$ there is $d_{j} \geq j$ and $f_{d_{j}} \in \operatorname{Hom}_{\hat{R}}\left(k, K_{d_{j}}\right)$ that cannot be extended to $E_{l} \rightarrow K_{d_{j}}$.

Each $E^{n}$ is an injective $\hat{R}$-module, therefore an injective $R$-module (by Lemma 7). So each of the complexes

$$
\begin{aligned}
& J_{d_{0}}=\ldots \rightarrow 0 \rightarrow 0 \rightarrow 0 \rightarrow E^{d_{0}} \stackrel{g_{d_{0}}}{\longrightarrow} E^{d_{0}+1} \rightarrow E^{d_{0}+2} \rightarrow \ldots \\
& J_{d_{1}}=\ldots \rightarrow 0 \rightarrow 0 \rightarrow E^{d_{1}-1} \rightarrow E^{d_{1}} \stackrel{g_{d_{1}}}{\longrightarrow} E^{d_{1}+1} \rightarrow E^{d_{1}+2} \rightarrow \ldots \\
& J_{d_{2}}=\ldots \rightarrow 0 \rightarrow E^{d_{2}-2} \rightarrow E^{d_{2}-1} \rightarrow E^{d_{2}} \stackrel{g_{d_{2}}}{\longrightarrow} E^{d_{2}+1} \rightarrow E^{d_{2}+2} \rightarrow \ldots
\end{aligned}
$$

is DG-injective (over $R$ ). By hypothesis, $\oplus_{i=0}^{\infty} J_{d_{i}}$ is DG-injective.

The same argument used in the proof of Proposition 3 shows that

$$
\frac{\Pi_{j=0}^{\infty} \operatorname{Ker} g_{d_{j}}}{\oplus_{j=0}^{\infty} \operatorname{Ker} g_{d_{j}}}=\frac{\prod_{j=0}^{\infty} K_{d_{j}}}{\oplus_{j=0}^{\infty} K_{d_{j}}}
$$

is an injective $R$-module, therefore an injective $\hat{R}$-module (by Lemma 7).

Then the same argument used in the proof of Proposition 4 shows that $f_{d_{j}}$ can be extended to a homomorphism $E_{l} \rightarrow K_{d_{j}}$ for infinitely many $j \geq 0$. Contradiction. So there is $d_{0} \geq 0$ such that for any $d \geq d_{0}$, any linear map $k \rightarrow K_{d}$ can be extended to $E_{l} \rightarrow K_{d}$.

By [5] Corollary 3.1.21, Soc $K_{d}=\operatorname{Soc} E_{\hat{R}}\left(K_{d}\right)=\operatorname{Soc} E(k)^{\beta_{d}}=k^{\beta_{d}}$. So $k^{\beta_{d}} \subset K_{d}, \forall d \geq 0$. Since $k^{\beta_{d}} \subset K_{d} \subset E(k)^{\beta_{d}}$ and any linear map $k \rightarrow K_{d}$ can be extended to $E_{l} \rightarrow K_{d}$, it follows that $E_{l}^{\beta_{d}} \subset K_{d} \Leftrightarrow\left\{x \in E(k)^{\beta_{d}} \mid\right.$ $\left.\hat{m}^{l} x=0\right\} \subset K_{d} \Leftrightarrow\left\{x \in E^{d} \mid \hat{m}^{l} x=0\right\} \subset K_{d}$ for any $d \geq d_{0}$.

The same argument used in the proof the Theorem 5 shows that for each $l \geq 1$ there is $\bar{n} \geq 1$ such that the entries $a_{i j}$ of the matrix that represents $\hat{f}_{n}$ are all in $\hat{m}^{l}$, for any $n \geq \bar{n}$. Since $a_{i j}$ are the entries of the matrix that represents $f_{n}$, we have $a_{i j} \in \hat{m}^{l} \cap R=m^{l}$ ([9], Corollaire pp. 26), for any $i \in\left\{1, \ldots, \beta_{n}\right\}, j \in\left\{1, \ldots, \beta_{n-1}\right\}$. 
Remark 1. Let $(R, m, k)$ be a commutative local noetherian ring such that every direct sum of DG-injective complexes of $R$-modules is DG-injective. If $\ldots \rightarrow R^{\beta_{1}} \stackrel{f_{1}}{\longrightarrow} R^{\beta_{0}} \stackrel{f_{0}}{\longrightarrow} k \rightarrow 0$ is a minimal projective resolution of $k$ then, by Theorem 6, for each $l \geq 1$ there is $n_{l} \geq 1$ such that the entries $a_{i j}$ $\left(1 \leq i \leq \beta_{n}, 1 \leq j \leq \beta_{n-1}\right)$ of the matrix that represents $f_{n}$ are all in $m^{l}$, for any $n \geq n_{l}$. It is not known if this condition guarantees the existence of an $\bar{n} \geq 1$ such that for any $n \geq \bar{n}$ all $a_{i j}$ are in $m^{l}$, for any $l \geq 1$, so all $a_{i j}$ are in $\cap_{l \geq 1} m^{l}=0$, that is $f_{n}=0$ for all $n \geq \bar{n}$, and so gl.dim $R=\operatorname{proj} \operatorname{dim} k<\infty$.

As a consequence of Theorem 6 we have that the class of DG-injective complexes over a commutative local artinian ring $R$ is closed under direct sums if and only if gl.dim $R<\infty$.

COROllary 1. Let $(R, m, k)$ be a commutative local artinian ring. The following are equivalent:

(1) Every direct sum of DG-injective complexes is DG-injective.

(2) gl.dim $R<\infty$.

PRoof. "(1) $\Rightarrow(2)$ " Since $R$ is artinian there is $l \geq 1$ such that $m^{l}=0$ ([5], Proposition 2.3.22).

Let $\ldots \rightarrow R^{\beta_{1}} \stackrel{f_{1}}{\longrightarrow} R^{\beta_{0}} \stackrel{f_{0}}{\longrightarrow} k \rightarrow 0$ be a minimal projective resolution of $k$. By Theorem 6 there is $n_{l} \geq 1$ such that the entries $a_{i j}$ of the matrix that represents $f_{n}$ are all in $m^{l}$, for any $n \geq n_{l}$. Hence $f_{n}=0$ for all $n \geq n_{l}$. Consequently proj $\operatorname{dim} k<\infty$. Since $R$ is local we have gl.dim $R=\operatorname{proj} \operatorname{dim} k$ ([11] Corollary 4.4.12). So gl.dim $R<\infty$.

(2) $\Rightarrow(1)$ Let $\left(J_{i}\right)_{i \in I}$ be a family of DG-injective complexes.

Since $R$ is noetherian and each $J_{i}^{n}$ is injective it follows that $\oplus_{i \in I} J_{i}^{n}$ is injective, $\forall n \geq 0$. Since gl.dim $R<\infty$ any complex of injective $R$-modules is DG-injective. So $\oplus_{i \in I} J_{i}$ is a DG-injective complex.

Theorem 7 below shows that the result holds for any commutative artinian ring.

THEOREM 7. Let $R$ be a commutative artinian ring. The following are equivalent:

(1) Every direct sum of DG-injective complexes of R-modules is DG-injective.

(2) gl.dim $R<\infty$.

Proof. This follows from the fact that any artinian ring is isomorphic to a product of local artinian rings. So then the result follows from the equivalence between the category of $R$-modules and the product of categories of modules over the local rings in the decomposition of $R$. 
Theorem 7 and the same argument as in the proof of Theorem 4 gives us the following result.

THEOREM 8. If $R$ is a commutative artinian ring then every direct sum of exact covers is an exact cover if and only if $\operatorname{gl} . \operatorname{dim} R<\infty$.

ACKNOWLEDGEMENT. I would like to thank Dr. Edgar Enochs for numerous advices and discussions while writing this paper.

\section{REFERENCES}

1. Avramov, L., and Foxby, H.-B., Homological dimensions of unbounded complexes, J. Pure Appl. Algebra 71 (1991), 129-155.

2. Bass, H., Injective dimension in noetherian rings, Trans. Amer. Math. Soc. 102 (1962), 189209.

3. Bass, H., On the ubiquity of Gorenstein rings, Math Z. 82 (1963), 8-28.

4. Chase, S., Direct product of modules, Trans. Amer. Math. Soc. 97 (1960), 457-473.

5. Enochs, E., and Jenda, O. M. G., Relative Homological Algebra, de Gruyter Exp. Math., 2000.

6. Enochs, E., Jenda, O. M. G., and Xu, J., Orthogonality in the category of complexes, Math. J. Okayama Univ. 38 (1996), 25-46.

7. Rotman, J. J., An Introduction to Homological Algebra, Academic Press, New York, 1979.

8. García Rozas, J. R., Covers and Envelopes in the Category of Complexes of Modules, CRC Press LLC, 1999.

9. Samuel, P., Progrès récents d'Algèbre locale, Instituto de Matemática Pura e Aplicada do Conselho Nacional de Pesquisas, 1959, Notas de Matemática.

10. Spaltenstein, N., Resolutions of unbounded complexes, Compositio Math. 65 (1988), 121154.

11. Weibel, C. A., An introduction to Homological Algebra, Cambridge Stud. Adv. Math. 38 (1994).

\footnotetext{
DEPARTMENT OF MATHEMATICS

UNIVERSITY OF KENTUCKY

LEXINGTON, KENTUCKY 40506-0027

USA

E-mail: iacob@ms.uky.edu.
} 